\title{
High-Sensitivity Troponin for Suspected Acute Coronary Syndrome in Patients With Chronic Kidney Disease Versus Patients Without Chronic Kidney Disease
}

\author{
David Clemons ${ }^{\mathrm{a}, \mathrm{b}}$, Aaron Lee ${ }^{\mathrm{a}, \mathrm{b}}$, Saaniya Ajmeria, ${ }^{\mathrm{a}}$, Vittorio Terrigno ${ }^{\mathrm{a}, \mathrm{b}}$, \\ Jordan Zaid ${ }^{\mathrm{a}, \mathrm{b}}$, Krystal Hunter ${ }^{\mathrm{c}}$, Satyajeet Roy ${ }^{\mathrm{a}, \mathrm{b}, \mathrm{d}}$ (1)
}

\begin{abstract}
Background: Heart disease is the leading cause of death in the United States. Patients with acute coronary syndrome (ACS) who have chronic kidney disease (CKD) have a twofold increase in mortality compared to patients with normal kidney function. Patients with $\mathrm{CKD}$ tend to have elevated baseline high-sensitivity cardiac troponin- T (hscTnT) levels. We studied patients with or without CKD to find out if a higher baseline hs-cTnT influenced the change in hs-cTnT (delta) when ruling in or ruling out ACS.
\end{abstract}

Methods: Eighty-nine patients were included in this study (29 with CKD; 60 without CKD). Delta hs-cTnT was dichotomized based on those who had delta of $\geq 5$, or $<5$. We calculated the positive predictive values, negative predictive values, sensitivities and specificities. Shapiro-Wilk test and independent $t$-test were used for the continuous variables. Mann-Whitney U test was used to examine the variables between the two groups. Chi-square test was used to compare the categorical variables between the two groups.

Results: The mean ages of patients with CKD and without CKD were 61.2 and 58.9 years, respectively $(\mathrm{P}=0.508)$. We found that although there were differences in the sensitivities, specificities, positive predictive values and negative predictive values of delta hs-cTnT $>5$ for ACS between the patients with CKD and without CKD, the differences were not statistically significant. Subgroup analysis showed that in patients with $\mathrm{CKD}$, the positive predictive values and sensitivities of delta hs-cTnT $>5$ for CAD requiring percutaneous coronary intervention (PCI) and stent were significantly higher compared to the patients without CKD (82.4\% vs. $27.3 \%$, and $82.4 \%$ vs. $40.0 \%$, respectively) $(\mathrm{P}<0.05)$.

Manuscript submitted April 29, 2021, accepted May 27, 2021

Published online June 25, 2021

aDepartment of Medicine, Cooper University Health Care, Camden, NJ, USA ${ }^{b}$ Cooper Medical School of Rowan University, Camden, NJ, USA

${ }^{\mathrm{c} C o o p e r}$ Research Institute, Cooper Medical School of Rowan University, Camden, NJ, USA

${ }^{\mathrm{d} C o r r e s p o n d i n g ~ A u t h o r: ~ S a t y a j e e t ~ R o y, ~ D e p a r t m e n t ~ o f ~ M e d i c i n e, ~ C o o p e r ~ U n i-~}$ versity Health Care, 1103 North Kings Highway, Suite 203, Cherry Hill, NJ, USA. Email: roy-satyajeet@cooperhealth.edu

doi: https://doi.org/10.14740/jocmr4515
Conclusions: In calculating delta hs-cTnT to rule in or rule out ACS, the presence of CKD does not influence the delta. Patients with CKD and a delta hs-cTnT $>5$ have significantly higher risk of undergoing PCI.

Keywords: Acute coronary syndrome; High sensitivity cardiac troponin-T; Coronary artery disease; Chronic kidney disease

\section{Introduction}

Heart disease is the leading cause of death for men and women in the United States [1]. Patients with acute coronary syndrome (ACS) who have chronic kidney disease (CKD) have a twofold increase in mortality compared to patients with ACS and normal kidney function [2]. The fifth generation cardiac troponin- $\mathrm{T}$ assay, also known as high-sensitivity cardiac troponin- $\mathrm{T}$ (hs-cTnT), is the most recent sensitive cardiac assay that has been approved by the United States Food and Drug Administration [3]. This test allows physicians to rapidly rule in or rule out patients for ACS [4]. Patients presenting to the emergency department with symptoms concerning of ACS can be ruled in or ruled out based on the 0 and $1 \mathrm{~h}$ blood samples [5]. This laboratory test allows healthcare workers to triage and safely manage patients in a timelier manner compared to the previous cardiac troponin assays [6]. Based on the hospital-specific cutoff values, if the first hs-cTnT happens to be below the cutoff and there has been a low suspicion for ACS, the patients can be ruled out within an hour of blood sample collection. If the initial hs-cTnT happens to be above the cutoff, a second hs-cTnT is drawn, and the change, or delta value (delta hs-cTnT), is calculated. A delta $>5$ raises a higher suspicion for ACS, and subsequently another hs-cTnT should be drawn $3 \mathrm{~h}$ later [5].

While following this protocol and making the clinical assessment of the patients with symptoms concerning ACS, healthcare providers must be aware of the different variables that may influence the hs-cTnT concentration. Factors, such as renal dysfunction, time from the onset of chest pain, and sex have been suggested as the factors which influence the baseline levels of hs-cTnT $[3,7]$. The kidneys play an integral role in metabolizing cardiac biomarkers, and patients with CKD 
may have difficulty with this process. It is well known that the patients with end-stage renal disease have an abnormally elevated baseline level of hs-cTnT compared to the patients who do not have CKD $[8,9]$.

In this study, we compared patients with and without CKD and analyzed whether higher baseline levels of hs-cTnT would influence the delta hs-cTnT when ruling in or ruling out patients who presented with symptoms of suspected ACS. We hypothesized that the presence of CKD would influence the delta hs-cTnT thereby impacting the diagnostic accuracy in predicting ACS.

\section{Materials and Methods}

\section{Study selection}

This study was a retrospective chart review of the existing electronic medical records of our patients who visited the emergency department for chest pain suspicious of ACS. We compared the differences in delta hs-cTnT and association of coronary artery disease (CAD) and percutaneous coronary intervention (PCI) in patients who presented with chest pain with or without CKD who were seen in our healthcare system between July 1, 2019 and December 31, 2019. The inclusion criteria were adult patients of age 18 years, or older, who presented with chest pain and had elevated hs-cTnT levels. The exclusion criteria were patients who were younger than 18 years of age, or who presented with other causes of chest pain, such as pulmonary embolism, pericarditis, aortic dissection, pleuritic, chest trauma, etc., as well as patients who were pregnant, or who had heart transplantation. The study was reviewed and approved by the Institutional Review Board of the Cooper University Health Care (CUHC), Camden, New Jersey, USA. This study was fully compliant with the ethical standards set forth by the CUHC Institutional Review Board.

\section{Data collection}

The following data were collected for each patient from the existing electronic medical records: age, gender, race, social history, comorbid medical conditions such as hypertension, diabetes mellitus, hyperlipidemia, hypothyroidism, other endocrine disorders, CAD, history of cardiac catheterization, history of stent placement, cerebral vascular accident or stroke, carotid artery stenosis, peripheral arterial disease, congestive heart failure, chronic obstructive pulmonary disease, asthma, CKD, liver disease, immunocompromised state, and cancer. We also collected the data on blood pressure, body mass index (BMI), lipid profile, estimated glomerular filtration rate (eGFR), white blood cell (WBC) count, serum hs-cTnT, hs-cTnT delta, use of medications such as non-steroidal anti-inflammatory drugs (NSAIDs), statin, angiotensin-converting enzyme inhibitor, diuretic, angiotensin II receptor blocker, beta-blocker, calcium channel blocker, and anticoagulant.

\section{Statistical analysis}

Collected data were entered into a Microsoft Excel (2016, Remond, Washington, USA) spreadsheet. Statistical analysis was done by using SPSS (Statistical Package for the Social Sciences, version 15.01, IBM, Armonk, New York, USA). A sample size estimation was not used for this study due to the limited number of patients who had a hs-cTnT test performed during the time frame of this study. Instead, the data of all patients who had the hs-cTnT test and the delta were reviewed who were seen in our healthcare system between July 1, 2019 and December 31, 2019.

The patients were divided into two groups. The first group represented the patients who had CKD stage IIIa, IIIb, IV, or $\mathrm{V}$; the second group represented patients without CKD. For the purpose of this study, CKD was defined as an eGFR value of 0 - $59 \mathrm{~mL} / \mathrm{min} / 1.73 \mathrm{~m}^{2}$, while an eGFR value of $60 \mathrm{~mL} / \mathrm{min} / 1.73$ $\mathrm{m}^{2}$ or greater was defined as no CKD. Patients who had severe $\mathrm{CAD}$ received coronary artery stents. In order to examine our hypothesis, the delta hs-cTnT was dichotomized based on those who had a delta value $\geq 5$, or $<5$. We then calculated the positive predictive values (PPVs), negative predictive values (NPVs), sensitivities and specificities. We used the continuous values of the hs-cTnT change to do a receiver operating characteristic (ROC) curve. In the secondary analysis of the continuous variables, we first tested their distribution by using the Shapiro-Wilk test with the plan to use independent $t$-tests if the test showed that the continuous variables were normally distributed. We used Mann-Whitney U test to examine the variables between the two groups if the data were not normally distributed. Chi-square test was used to compare the categorical variables between the two groups. Logistic regression was used to examine multiple factors that contribute to have a delta hs-cTnT of $>5$. In this study, significance was defined as a $\mathrm{P}<0.05$.

\section{Results}

A total of 89 patients were included in this study: 29 (32.6\%) with CKD and $60(67.4 \%)$ without CKD. The mean ages of patients with CKD and without CKD were 61.2 and 58.9 years, respectively. There was no statistically significant difference in the mean ages between the two groups (Table 1). In the CKD group, the frequencies of Whites, Blacks and Hispanics were $44.8 \%, 34.5 \%$ and $20.7 \%$, respectively; while the race frequencies were $60 \%, 20 \%$ and $20 \%$, respectively in the group without CKD. There was no statistically significant difference in the race distribution between the two groups (Table 1). About $34.5 \%$ of the patients in the CKD group and $44.1 \%$ in the group without CKD used tobacco products. Similarly, $20.7 \%$ of the patients in the CKD group and $27.1 \%$ in the group without CKD used alcoholic beverages (Table 1). The differences in the tobacco use and alcohol consumption were not statistically significant between the two groups. The mean systolic blood pressure of the patients in the CKD group was significantly higher than the group without CKD (135 vs. 125 $\mathrm{mm} \mathrm{Hg})(\mathrm{P}<0.05)$. There was no significant difference in the mean diastolic pressure of the patients between the two groups. 
Table 1. Baseline Characteristics, Comorbidities and Medications

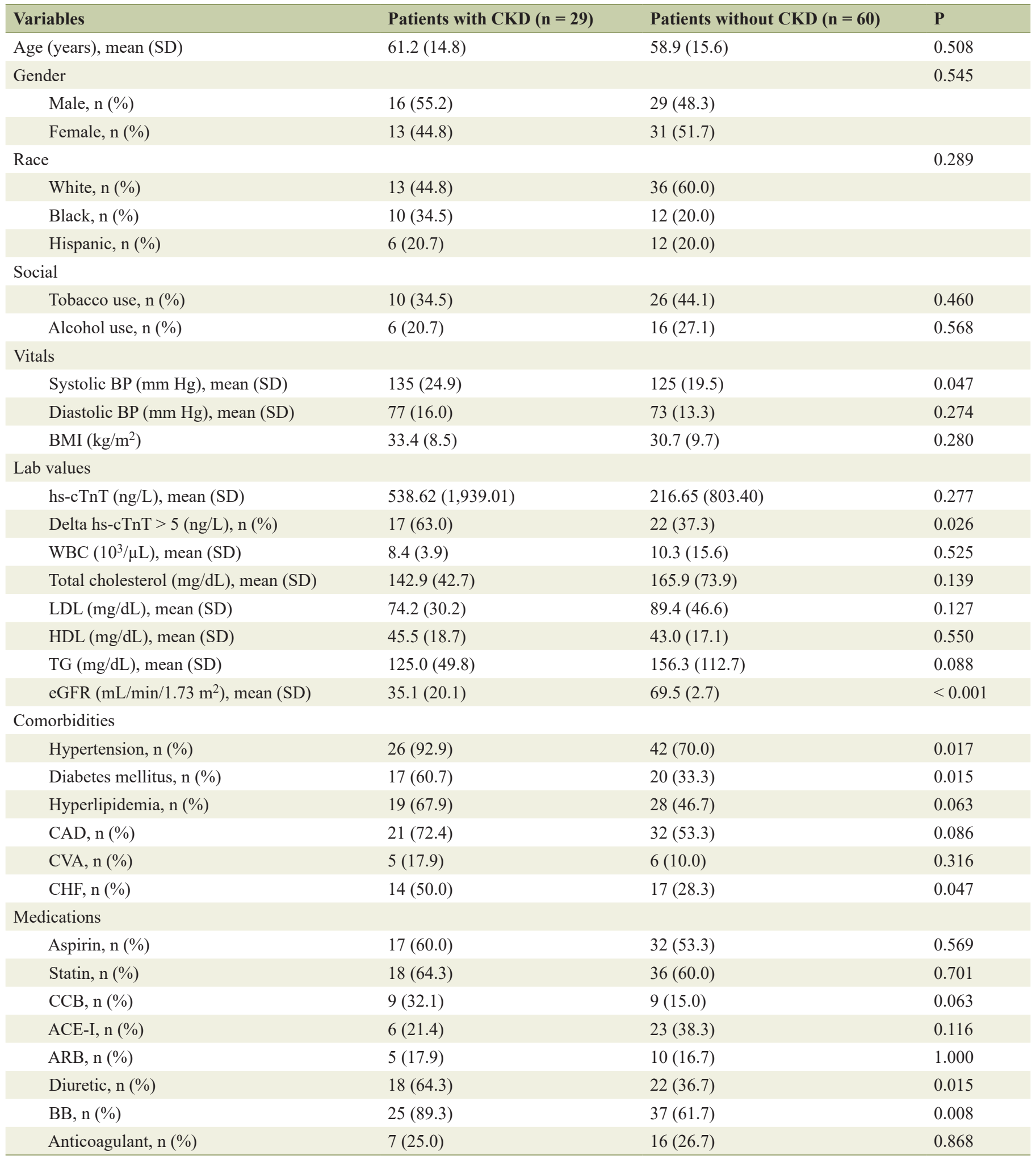

CKD: chronic kidney disease; SD: standard deviation; BP: blood pressure; BMI: body mass index; hs-cTnT: high-sensitivity cardiac troponin-T (0 h value); WBC: white blood cell; LDL: low-density lipoprotein; HDL: high-density lipoprotein; TG: triglycerides; eGFR: estimated glomerular filtration rate; CAD: coronary artery disease; CVA: cerebrovascular accident; CHF: congestive heart failure; CCB: calcium channel blocker; ACE-I: angiotensin-converting enzyme inhibitor; ARB: angiotensin II receptor blocker; BB: beta-blocker. 


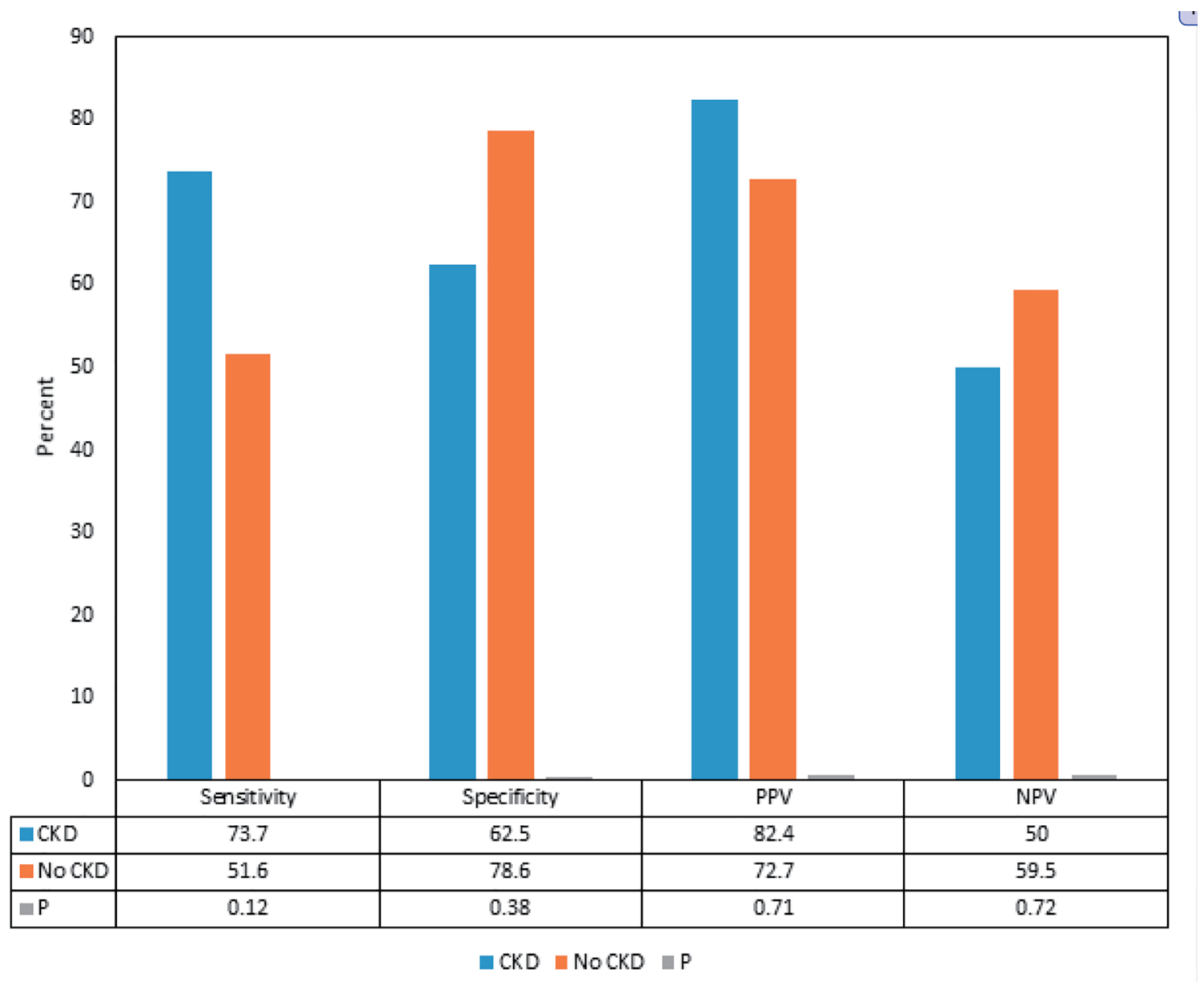

Figure 1. Comparison of sensitivities, specificities, positive predictive values and negative predictive values of delta hs-cTnT > 5 for acute coronary syndrome and coronary artery disease in patients with and without chronic kidney disease. CKD: chronic kidney disease; PPV: positive predictive value; NPV: negative predictive value; hs-cTnT: high-sensitivity cardiac troponin-T.

Although the mean BMI of the patients in the CKD group was higher than the patients without CKD $(33.4 \pm 8.5$ vs. $30.7 \pm$ 9.7), the difference was not statistically significant (Table 1). The mean eGFR of the patients in the CKD group was significantly lower than the group without CKD $(35.1 \pm 20.1$ vs. 69.5 $\left.\pm 2.7 \mathrm{~mL} / \mathrm{min} / 1.73 \mathrm{~m}^{2}\right)(\mathrm{P}<0.001)$. The frequency of delta hscTnT $>5 \mathrm{ng} / \mathrm{L}$ was significantly higher in the CKD group than the group without CKD $(63.0 \%$ vs. $37.3 \%)(\mathrm{P}<0.05)$ (Table 1). There were no significant differences in the mean hs-cTnT, WBC count, total cholesterol, low-density lipoprotein (LDL) cholesterol, high-density lipoprotein (HDL) cholesterol and triglyceride levels between the two groups (Table 1).

Hypertension, CAD, hyperlipidemia, diabetes mellitus and congestive heart failure were the top five associated comorbid medical conditions in the patients with CKD group as well as in the group of patients without CKD (Table 1). Patients in the CKD group were found to have significantly higher frequencies of comorbid medical conditions, such as hypertension, diabetes mellitus, congestive heart failure, and liver disease compared to the patients without CKD $(92.9 \%$ vs. $70.0 \%, 60.7 \%$ vs. $33.3 \%, 50.0 \%$ vs. $28.3 \%$, and $7.1 \%$ vs. $0.0 \%$, respectively) $(\mathrm{P}<0.5)$ (Table 1). There were no significant dif- ferences in the frequencies of other comorbidities between the two groups, such as hyperlipidemia, hypothyroidism, CAD, cerebrovascular disease, peripheral vascular disease, carotid artery stenosis, chronic obstructive pulmonary disease, asthma, immunocompromised state, or cancer (Table 1).

There was no statistically significant difference in the use of medications, such as aspirin, NSAID, statin, calcium channel blocker, angiotensin-converting enzyme inhibitor, and angiotensin II receptor blocker, between the two groups. The use of beta-blocker and diuretic was significantly higher in the patients with CKD compared to the patients without CKD $(64.3 \%$ vs. 36.7 , and $89.3 \%$ vs. $61.7 \%)(\mathrm{P}<0.05)($ Table 1$)$.

We found that although there were differences in the sensitivities, specificities, PPVs and NPVs of delta hs-cTnT $>5$ for ACS between the patients with CKD and without CKD, the differences were not statistically significant (Fig. 1). Subgroup analysis showed that in patients with CKD, the PPVs and sensitivities of delta hs-cTnT $>5$ for CAD requiring PCI and stent were significantly higher compared to the patients without CKD $(82.4 \%$ vs. $27.3 \%$, and $82.4 \%$ vs. $40.0 \%$, respectively) $(\mathrm{P}<0.05)$ (Fig. 2 ).

Logistic regression analysis showed significant findings only for race and hypertension (Table 2). In comparison to the 


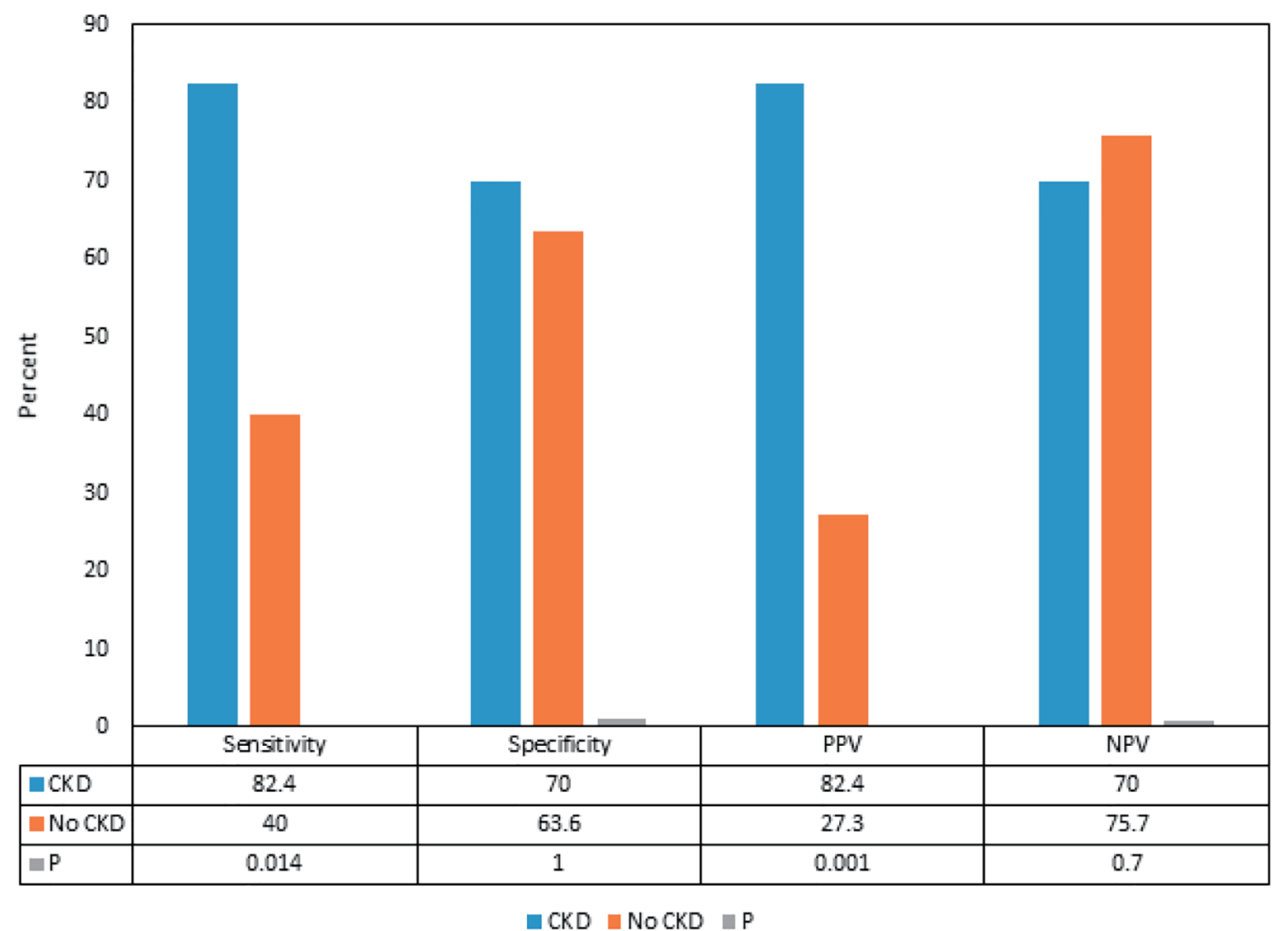

Figure 2. Comparison of sensitivities, specificities, positive predictive values and negative predictive values of delta hs-cTnT > 5 for acute coronary syndrome and coronary artery disease requiring coronary stents in patients with and without chronic kidney disease. CKD: chronic kidney disease; PPV: positive predictive value; NPV: negative predictive value; hs-cTnT: high-sensitivity cardiac troponin-T.

Table 2. Influence of Risk Factors on Delta hs-cTnT > 5

\begin{tabular}{llllll}
\hline Risk factor & B & P & Exp(B) & \multicolumn{2}{c}{ 95\% CI for Exp(B) } \\
\cline { 4 - 6 } Age (years) & 0.040 & 0.088 & 1.041 & 0.994 & 1.090 \\
Sex male & 0.802 & 0.188 & 2.230 & 0.675 & 1.134 \\
White vs. Black & 1.544 & 0.033 & 4.683 & 0.434 & 19.339 \\
White vs. Hispanic & 0.558 & 0.433 & 1.747 & 0.607 & 7.038 \\
Tobacco use & 0.741 & 0.241 & 2.098 & 0.164 & 7.249 \\
Alcohol use & -0.487 & 0.469 & 0.615 & 1.031 & 2.298 \\
Hypertension & 1.991 & 0.047 & 7.323 & 0.243 & 52.037 \\
Diabetes mellitus & -0.258 & 0.662 & 0.773 & 0.165 & 2.456 \\
Hyperlipidemia & -0.513 & 0.436 & 0.599 & 0.612 & 2.175 \\
CAD & 0.855 & 0.213 & 2.351 & 0.248 & 9.030 \\
CVA & 0.329 & 0.708 & 1.390 & 0.384 & 7.793 \\
CHF & 0.242 & 0.693 & 1.273 & 0.573 & 4.219 \\
CKD & 0.633 & 0.297 & 1.884 & & 6.197 \\
\hline
\end{tabular}

hs-cTnT: high-sensitivity cardiac troponin-T; Cl: confidence interval; CAD: coronary artery disease; CVA: cerebrovascular accident; CHF: congestive heart failure; CKD: chronic kidney disease. 
White race, Black race had 4.68 times greater odds of having hs-cTnT delta $>5$ (95\% confidence interval (CI) 1.03 - 19.34) $(\mathrm{P}=0.033)$. Similarly, subjects with hypertension had 7.32 times greater odds of having hs-cTnT delta $>5$ (95\% CI 1.03 - 52.04) $(\mathrm{P}=0.047)$. We found that CKD was not a factor in having hs-cTnT delta $>5$ (odds ratio (OR) 1.88, 95\% CI 0.57 - 6.19; $\mathrm{P}=0.297$ ) (Table 2).

\section{Discussion}

The influence of CKD on the hs-cTnT, and on its interpretation in predicting ACS as per the delta hs-cTnT algorithm in our study showed that although there were differences in the sensitivities, specificities, PPVs and NPVs of delta hs-cTnT $>$ 5 for ACS between the patients with CKD and without CKD, the differences were not statistically significant. Interestingly, we found that the PPV and sensitivity of delta hs-cTnT $>5$ for $\mathrm{CAD}$ requiring $\mathrm{PCI}$ and stent placement were significantly higher in patients with $\mathrm{CKD}$ compared to the patients without CKD.

CKD happens to be one of the commonly known variables that can affect the levels of hs-cTnT. As per the analysis by Januzzi, the frequency of elevated cardiac troponins (cTn) in asymptomatic CKD depends on the assay (cTnT $>\mathrm{cTnI})$ and the cutoff values [10]. This limitation influences the specificity of hs-cTnT for ACS in patients with CKD, in whom it tends to be lower compared with patients without CKD [10]. In our study we found that the sensitivity of delta hs-cTnT $>$ 5 for ACS was greater in patients with CKD compared to the patients without CKD, but the difference was not statistically significant. The incidence and prevalence of ACS and CAD is known to be high in patients with CKD which has been believed to make the interpretation even more challenging, but our study suggests that delta hs-cTnT is not influenced by the presence or absence of CKD.

A study from Sarnak and associates reported that the cardiovascular mortality in patients with advanced renal failure is 10 - 30 times higher than in the general population [11]. Another study performed by Levey and associates concluded that patients with CKD should be considered an extremely high risk group for the future development of cardiovascular disease. The study went on to delineate that the patients with CKD have an increased prevalence of both CAD and left ventricular hypertrophy (LVH), which increases their risk of cardiovascular death [12]. Our subgroup analysis was consistent with these studies as we found that the PPV and sensitivity of delta hs-cTnT $>5$ for CAD requiring PCI and stent placement were significantly higher in patients with CKD compared to the patients without CKD, supporting the higher association of CAD in patients with CKD. It is, therefore, suggested that while the elevated hs-cTnT in CKD leads to a higher risk for false positive diagnosis of ACS, it is necessary to take this finding seriously in patients with CKD as true positive results are associated with a heightened mortality risk compared to the patients without CKD [13]. It is also important to understand that the elevated level of hs-cTnT in asymptomatic patients with CKD is associated with an increase in the incidence of
ACS which also increases the risk of mortality by two- to fivefold $[14,15]$. Hence, serial measurements and accurate documentation of delta hs-cTnT offers the best possible interpretation of the hs-cTnT.

A single center study performed by Gunsolus and associates found that renal dysfunction affected testing results for hs-cTnT as a rule-in test, but did not affect its ability to be used as a test to rule out ACS [7]. Our study did not find this correlation, instead we found no statistical difference between our two patient populations when it came to ruling in or ruling out ACS.

Although the risk factors of CKD and CAD tend to correlate well, but CKD by itself offers increased risk of CAD through several systemic changes. A study performed by Liu and associates hypothesized that protein bound uremic toxins (PBUTs), indoxyl sulfate (IS) in particular, play a major role in the progression of cardiovascular disease in patients with CKD [16]. A study by Gryp and associates examined the accumulation of PBUTs in patients with CKD [17]. They specifically examined the amount of gut elimination of these products in patients with CKD. However, they did not find an increased gut excretion of PBUTs in patients with CKD, which led them to hypothesize that the increased levels of PBUTs occur in patients with worsening renal function [17]. It is noteworthy to know that the PBUTs are not adequately cleared during hemodialysis, hence imposing even higher risk of CAD.

An animal study performed by Lekawanvijit and associates found that reducing IS levels helped to decrease levels of cardiorenal fibrosis [18]. They concluded that the IS contributed to cardiorenal syndrome. Another mechanism that may cause cardiac dysfunction in patients with CKD was postulated by Liu and colleagues. They proposed that FGF23, a growth factor that is increased in patients with $\mathrm{CKD}$, may play a role in the development of LVH and vascular calcification [16]. These mechanisms may similarly influence the renal excretion of hs-cTnT which may explain the significantly higher frequency of delta hs-cTnT $>5 \mathrm{ng} / \mathrm{L}$ in the CKD group in our study compared to the group without CKD.

While we acknowledge that hs-cTnT is not the only factor to examine when considering whether a patient warrants cardiac catheterization, it is an important part of our institution's ACS workup. Other variables in interpreting delta hscTnT in patients with suspected ACS include patients' age, risk factors for $\mathrm{CAD}$, clinical suspicion based on current symptoms, electrocardiographic changes, and previous medical history. All of these factors play a role in the determination of which patients will undergo cardiac catheterization for suspected ACS.

In this study, our findings of significantly higher association of hypertension and higher mean systolic blood pressure in patients in the CKD group compared to the group without CKD aligns with several studies that have found similarly higher associations of hypertension in CKD, and also concluded that hypertension in CKD is predominantly systolic [19-21]. It has been well documented that hypertension and CKD are closely interlinked pathophysiologic states. Persistent systolic hypertension leads to progressive decline in the kidney function and worsens $\mathrm{CKD}$ which in turn leads to worsening blood pressure 
control [22]. Additionally, our patients with CKD had significantly higher association of diabetes compared to the patients without CKD. According to the Centers for Disease Control and Prevention, diabetes remains a leading cause of CKD and about one-third of adults with diabetes have CKD [23].

Among other comorbid conditions, we found that the patients in the CKD group had significantly higher association of congestive heart failure and liver disease compared to the patients without CKD. With progression of renal function deterioration, the prevalence of congestive heart failure increases greatly, which can reach up to $65-70 \%$ with the advancement of CKD to end-stage renal disease [24]. About one-quarter of patients with CKD develop congestive heart failure which is mostly secondary to ischemic heart disease [24]. Hepatic involvement in patients with CKD has been attributed secondary to a multitude of pathophysiological changes and an interdependence between the immune system of the liver, which is considered as a lymphoid organ, as well as the various immunopathogenic involvement of the kidneys [25].

Our study brings a unique perspective in the interpretation of delta hs-cTnT in the assessment of ACS in patients with or without CKD. On the one hand, we found that the delta hscTnT was not influenced by the presence or absence of CKD in predicting presence of ACS, while on the other hand, delta hs-cTnT offered a significantly higher PPV and sensitivity for the need for PCI and coronary stent placement in patients with CKD.

There were a few limitations in our study. Being a retrospective chart review of the information available in our electronic medical record, we had to fully rely on the documentations made by the patient care teams. On the other hand, the major strength of our study was the availability of accurately documented data of the patients after presentation in the emergency room, including the presenting symptoms, risk factors, serial hs-cTnT measurements, and the steps taken during the management based on the standard ACS protocol set forth by our institution, which allowed us to review the documentation of the course and outcome of each patient included in our study.

\section{Conclusion}

In calculating delta hs-cTnT to rule in or rule out ACS, the presence of CKD does not influence the delta. Patients with CKD and a delta hs-cTnT $>5$ have significantly higher risk of undergoing PCI.

\section{Acknowledgments}

The authors thank Christine Rickette, RN (study coordinator) for her contribution to this study.

\section{Financial Disclosure}

None to declare.

\section{Conflict of Interest}

None to declare.

\section{Informed Consent}

Not applicable. Being a retrospective chart review study the Institutional Review Board waived the need for informed consent.

\section{Author Contributions}

DC and SR made substantial contributions to the study design, drafting, data acquisition and analysis, and manuscript writing. AL, SA, VT and JZ contributed in data collection and manuscript writing. $\mathrm{KH}$ analyzed the data. SR contributed in revising the manuscript critically for improved intellectual content, and final approval for the version to be published.

\section{Data Availability}

The authors declare that data supporting the findings of this study are available within the article.

\section{References}

1. Centers for Disease Control and Prevention, National Center for Health Statistics. Underlying Cause of Death 1999-2019 on CDC WONDER Online Database, released in 2020. Data are from the Multiple Cause of Death Files, 1999-2019, as compiled from data provided by the 57 vital statistics jurisdictions through the Vital Statistics Cooperative Program. Accessed on Apr 24, 2021, at: http:// wonder.cdc.gov/ucd-icd10.html.

2. Marenzi G, Cabiati A, Assanelli E. Chronic kidney disease in acute coronary syndromes. World J Nephrol. 2012;1(5):134-145.

3. Rubini Gimenez M, Badertscher P, Twerenbold R, Boeddinghaus J, Nestelberger T, Wussler D, Miro O, et al. Impact of the US Food and Drug Administration-approved sex-specific cutoff values for high-sensitivity cardiac troponin $\mathrm{t}$ to diagnose myocardial infarction. Circulation. 2018;137(17):1867-1869.

4. Christenson RH, Mullins K, Duh SH. Validation of highsensitivity performance for a United States Food and Drug Administration cleared cardiac troponin I assay. Clin Biochem. 2018;56:4-10.

5. Twerenbold R, Boeddinghaus J, Mueller C. Update on high-sensitivity cardiac troponin in patients with suspected myocardial infarction. Eur Heart J Suppl. 2018;20:G2G10.

6. Reichlin T, Schindler C, Drexler B, Twerenbold R, Reiter M, Zellweger C, Moehring B, et al. One-hour ruleout and rule-in of acute myocardial infarction using 
high-sensitivity cardiac troponin T. Arch Intern Med. 2012;172(16):1211-1218.

7. Gunsolus I, Sandoval Y, Smith SW, Sexter A, Schulz K, Herzog CA, Apple FS. Renal dysfunction influences the diagnostic and prognostic performance of high-sensitivity cardiac troponin I. J Am Soc Nephrol. 2018;29(2):636643.

8. Khan NA, Hemmelgarn BR, Tonelli M, Thompson CR, Levin A. Prognostic value of troponin $\mathrm{T}$ and I among asymptomatic patients with end-stage renal disease: a meta-analysis. Circulation. 2005;112(20):3088-3096.

9. Tarapan T, Musikatavorn K, Phairatwet P, Takkavatakarn K, Susantitaphong P, Eiam-Ong S, Tiranathanagul K. High sensitivity Troponin-I levels in asymptomatic hemodialysis patients. Ren Fail. 2019;41(1):393-400.

10. Januzzi Jr J. Causes of Non ACS related troponin elevations. American College of Cardiology. 2010. Accessed on Apr 24, 2021, at: https://www.acc.org/latest-in-cardiology/articles/2014/07/18/13/16/causes-of-non-acs-related-troponin-elevations.

11. Sarnak MJ, Levey AS, Schoolwerth AC, Coresh J, Culleton B, Hamm LL, McCullough PA, et al. Kidney disease as a risk factor for development of cardiovascular disease: a statement from the American Heart Association Councils on kidney in cardiovascular disease, high blood pressure research, clinical cardiology, and epidemiology and prevention. Hypertension. 2003;42(5):1050-1065.

12. Levey AS, Beto JA, Coronado BE, Eknoyan G, Foley $\mathrm{RN}$, Kasiske BL, Klag MJ, et al. Controlling the epidemic of cardiovascular disease in chronic renal disease: what do we know? What do we need to learn? Where do we go from here? National Kidney Foundation Task Force on Cardiovascular Disease. Am J Kidney Dis. 1998;32(5):853-906.

13. Aviles RJ, Askari AT, Lindahl B, Wallentin L, Jia G, Ohman EM, Mahaffey KW, et al. Troponin T levels in patients with acute coronary syndromes, with or without renal dysfunction. N Engl J Med. 2002;346(26):20472052.

14. Troyanov S, Ly QH, Schampaert E, Ammann H, Lalumiere G, Madore F, Querin S. Diagnostic specificity and prognostic value of cardiac troponins in asymptomatic chronic haemodialysis patients: a three year prospective study. Heart. 2005;91(9):1227-1228.

15. Apple FS, Murakami MM, Pearce LA, Herzog CA. Predictive value of cardiac troponin $\mathrm{I}$ and $\mathrm{T}$ for subsequent death in end-stage renal disease. Circulation. 2002;106(23):2941-2945.

16. Liu M, Li XC, Lu L, Cao Y, Sun RR, Chen S, Zhang PY. Cardiovascular disease and its relationship with chronic kidney disease. Eur Rev Med Pharmacol Sci. 2014;18(19):2918-2926.

17. Gryp T, De Paepe K, Vanholder R, Kerckhof FM, Van Biesen W, Van de Wiele T, Verbeke F, et al. Gut microbiota generation of protein-bound uremic toxins and related metabolites is not altered at different stages of chronic kidney disease. Kidney Int. 2020;97(6):1230-1242.

18. Lekawanvijit S, Kumfu S, Wang BH, Manabe M, Nishijima F, Kelly DJ, Krum H, et al. The uremic toxin adsorbent AST-120 abrogates cardiorenal injury following myocardial infarction. PLoS One. 2013;8(12):e83687.

19. Andersen MJ, Khawandi W, Agarwal R. Home blood pressure monitoring in CKD. Am J Kidney Dis. 2005;45(6):994-1001.

20. Agarwal R. Systolic hypertension in hemodialysis patients. Semin Dial. 2003;16(3):208-213.

21. Agarwal R, Andersen MJ. Correlates of systolic hypertension in patients with chronic kidney disease. Hypertension. 2005;46(3):514-520.

22. $\mathrm{Ku} \mathrm{E}$, Lee BJ, Wei J, Weir MR. Hypertension in CKD: core curriculum 2019. Am J Kidney Dis. 2019;74(1):120131.

23. Centers for Disease Control and Prevention. Chronic kidney disease in the United States, 2019. Atlanta, GA: US Department of Health and Human Services. Centers for Disease Control and Prevention; 2019. Accessed on Apr 24, 2021, at: https://www.cdc.gov/kidneydisease/publications-resources/annual-report/ckd-risk-prevention.html.

24. Silverberg D, Wexler D, Blum M, Schwartz D, Iaina A. The association between congestive heart failure and chronic renal disease. Curr Opin Nephrol Hypertens. 2004;13(2):163-170.

25. Gluhovschi G, Petrica L, Sporea I, Timar R, Curescu M, Velciov S, Gluhovschi C. Chronic kidney disease - chronic liver disease. An immunologic cross-talk. Rom J Intern Med. 2015;53(1):3-12. 